\title{
Energy efficiency of a solar domestic hot water system
}

\author{
Miroslaw Zukowski ${ }^{1, *}$ \\ ${ }^{1}$ Bialystok University of Technology, Department of HVAC Engineering, ul. Wiejska 45E, \\ 15-351 Bialystok, Poland
}

\begin{abstract}
The solar domestic hot water (SDHW) system located on the campus of Bialystok University of Technology is the object of the research described in the current paper. The solar thermal system is composed of 35 flat plate collectors, 21 evacuated tube collectors and eight hot water tanks with the capacity of $1 \mathrm{~m}^{3}$ of each. Solar facility is equipped with hardware for automatic data collection. Additionally, the weather station located on the roof of the building provides measurements of basic parameters of ambient air and solar radiation. The main objective of Regional Operational Program was the assessment of the effectiveness of this solar energy technology in the climatic conditions of the north-eastern Poland. Energy efficiency of SDHW system was defined in this research as the ratio between the useful heat energy supplied to the domestic hot water system and solar energy incident on the surface of solar panels. Heat loss from water storage tanks, and from the pipe network to the surrounding air, as well as the electrical energy consumed by the pumps have been included in the calculations. The paper presents the detailed results and conclusions obtained from this energy analysis.
\end{abstract}

\section{Introduction}

Economic assessment of energy-conversion systems is quite complex. The long-term energy efficiency is the key factor of this analysis. Experimental studies carried out on the existing and operating facilities will allow to develop the energy balance of the whole solar system in a very accurate way. Unfortunately, this procedure is quite expensive. As it turned out, the additional cost of the monitoring and automatic data collection (ADC) of operating parameters of the solar domestic hot water (SDHW) system tested by the author, was equal about $2 / 3$ of the cost of materials and installation of the solar system. For this reason, experimental studies to evaluate the energy efficiency of medium and large solar installations are not often performed.

A large number of actual data from field-testing and monitoring of domestic solar hot water systems in China have been analysed by Yao et al. [1]. They discovered that there are many problems resulting from the relatively large amount of measurement error and use of different methodologies for assessing and monitoring. The authors proposed the methods and indicators for precise estimation of solar hot water systems.

\footnotetext{
*Corresponding author: m.zukowski@.pb.edu.pl
} 
The results of evaluation of water heating system supported by $640 \mathrm{~m}^{2}$ of solar collectors installed on high-rise housing complex in South Korea have been presented by Yoo in [2]. Unfortunately, as it turned out, the total energy gains from three years of SDHW operation is lower by a half of the design assumptions. A wide gap between theory and practice can be observed in this case. Analysis of measurement results have shown that the collector efficiency ranges from $42 \%$ in August to $53 \%$ in April. Unfortunately, the author has not performed an assessment of the energy efficiency of this large solar facility.

A solar domestic hot water system installed on a one-family house in Dublin (Ireland) has been tested by Gill et al. [3]. Based on the analysis of the annual results of experimental research the overall efficiency of the solar system has been estimated at about $63 \%$. It should be noted that the key components of heat loss have been taken into account by the authors of this study.

Several studies have investigated the energy and economic efficiency of the whole SDHW system using some simplifications. For example, solar collectors are treated as the only part of the installation that generates heat loss. Some selected studies of this kind are presented below.

Stevanovic and Pucar [4] have estimated the performance of solar water heating system with the f-Chart method. The results of theoretical analysis have been used to determine the level of the subsidy should be offered by the Serbian government to increase the popularity of renewable energy sources (RES) in this country.

The energetic, economic and environmental impacts of different types of water heating systems for the USA typical residential buildings located in Los Angeles, Atlanta, and Chicago have been investigated by Hang et al. [5]. Unfortunately, the performance of solar water heating systems has been determined only based on the thermal characteristics of the flat-plate (efficiency - 55\%), and evacuated-tube (efficiency - 59\%) solar collectors provided by the manufacturer.

Lin et al. [6] have developed a procedure for estimating the payback period of residential SDHW system. Daily solar radiation and hot water consumption pattern per person have been taken into account in the calculation of annual energy gains for case study in southern Taiwan. The analysis has assumed that the thermal efficiency of the collectors (do not exceeding $40 \%$ ) is equivalent to the performance of the whole system.

Fifteen different energy supply systems for single family houses in the northern European climate conditions have been analysed by Dziugaite-Tumeniene et al. [7]. The theoretical analysis made by the authors has been based on the integrated assessment model of an energy supply system for an energy-efficient building (ESSINTEGRA). Using this method has allowed to obtain energy efficiency (equal to $31 \%$ ) of the system consisting of the wood boiler and solar collectors.

Based on the literature review, it can be concluded that there are not much information on the research projects related to the experimental evaluation of the energy efficiency of medium solar systems used for domestic water heating. Thus, the author hopes that this paper will contribute to increase the knowledge on this important issue and provide the necessary data to design and economic analysis of SDHW systems.

\section{Description of the research object}

Regional Operational Program of Podlaskie Voivodeship on the practical application of renewable energy technologies were being implemented at the Bialystok University of Technology (BUT) in the years 2013-2015. One of the practical results of this project was installation of the solar domestic hot water system (SDHW). Solar facility is located on the roof of the Hotel for Research Assistants on BUT campus in Bialystok (Fig. 1). The solar thermal system is composed of two parallel-connected subsystems: the first includes of 
35 flat plate collectors (FPCs), connected in series of 5 units in one section, with a total area $A_{F P C}$ of about $72 \mathrm{~m}^{2}\left(65.5 \mathrm{~m}^{2}\right.$ - aperture area), and the second consists 21 evacuated tube collectors (ETCs), connected in series of 3 units in one section, with a total area $A_{E T C}$ of more than $74 \mathrm{~m}^{2}\left(67 \mathrm{~m}^{2}\right.$ - active area). Eight tanks with the total capacity of about $8 \mathrm{~m}^{3}$ are used to store the hot water heated by the solar thermal panels.

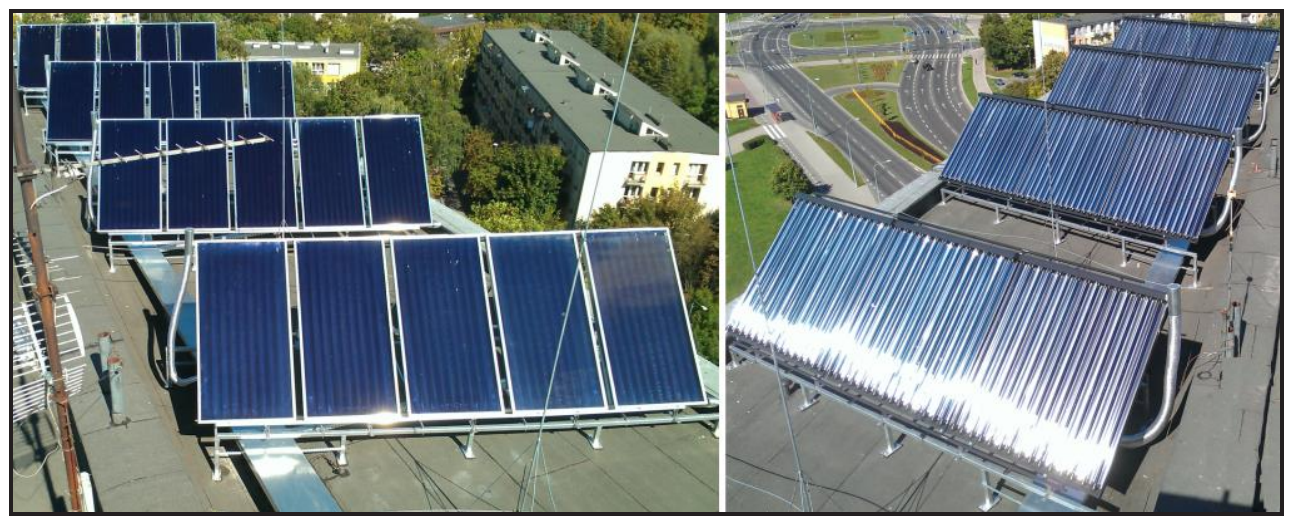

Fig. 1. Flat plate solar collectors connected in series of 5 units in one section (left side of the fig.), and evacuated tube solar collectors connected in series of 3 units in one section (right side of the fig.).

Solar facility is equipped with hardware for automatic data collection. The following elements are included in the data capture system: 17 heat meters, 4 electricity meters used for recording electric energy consumption of pumps, and 42 PT 500 platinum resistance temperature sensors for monitoring of the temperature distribution in the whole system. Additionally, the weather station located on the roof of the hotel provides measurements of the basic parameters of ambient air and solar radiation. All the data collected by the ADC system are readily available via the Internet. The simplified scheme of the hydraulic circuits of the solar system are shown in Fig. 2.

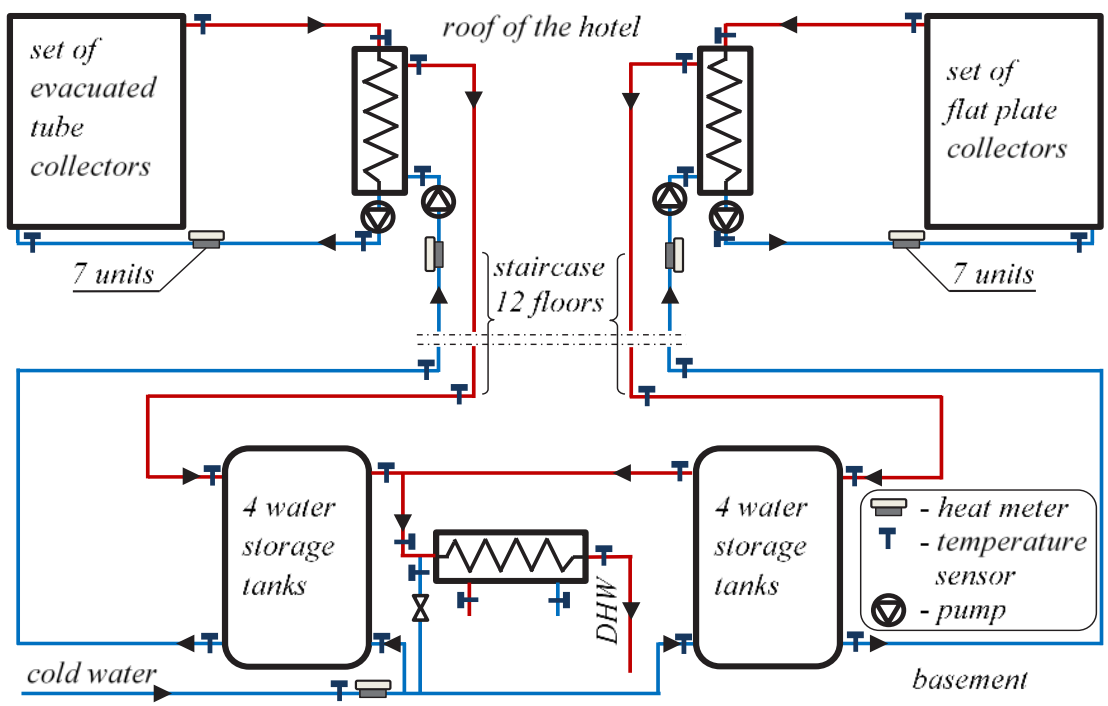

Fig. 2. Simplified diagram of the SDHW system with the location of measuring instruments and sensors. 


\section{Energy efficiency of a solar domestic hot water system}

The main objective of the previously mentioned Regional Operational Program was the assessment of the effectiveness of solar water heating technology in the climatic conditions of the north-eastern Poland. Energy efficiency of SDHW system $\eta_{S Y S}$ has been defined in this research based on Eq. (1) as the difference between the useful heat energy supplied to the tap water system in the hotel $E_{D H W}$ and electrical energy consumed by the pumps $E_{P}$ divided by solar energy incident on the solar panels $E_{S O L}$.

As mentioned earlier, ultrasonic heat meters have been utilised to obtain the value of thermal energy in 17 points at the system. Relative measurement error of these devices is small, and according to the manufacturer's information, is only $2 \%$. The data recorded by heat meters have been used to prepare the energy balance. The study covered the period from 1 May 2015 to 30 November 2016.

$$
\begin{gathered}
\eta_{S Y S}=\frac{E_{D H W}-E_{P}}{E_{S O L}} \times 100 \\
E_{D H W}=\sum_{1}^{n m}\left[m_{D H W} \cdot n d \cdot 24 \cdot 3600 \cdot\left(c_{p} \cdot\left(T_{F P C}-T_{C W}\right)+c_{p} \cdot\left(T_{E T C}-T_{C W}\right)\right)\right] \\
E_{S O L}=\sum_{1}^{n m} q_{S O L} \cdot n d \cdot 24 \cdot 3600 \cdot\left(A_{F P C}+A_{E T C}\right)
\end{gathered}
$$

where: $n m$ - number of months, $m_{D H W}-\mathrm{DHW}$ mass flow rate $[\mathrm{kg} / \mathrm{s}], n d$ - number of days in a month, $c_{p}$ - heat capacity of water $[\mathrm{J} / \mathrm{kg} / \mathrm{K}], T_{F P C}$ - temperature of the water flowing from the tanks supplied by FPCs $[\mathrm{K}], T_{C W}$ - temperature of the cold water $[\mathrm{K}]$, $T_{E T C}$ - temperature of the water flowing from the tanks supplied by ETCs $[\mathrm{K}], q_{S O L}-$ solar radiation flux that is incident on the collector surface $\left[\mathrm{W} / \mathrm{m}^{2}\right]$.

The weather station is located very close to the collectors and total solar radiation sensor is set at the same angle as the panels i.e. $40^{\circ}$. The sampling frequency of the weather parameters is equal to 5 seconds, and other parameters of the system are collected in oneminute time steps. Therefore, it was necessary to average all the results of measurement on a one hour interval. Then, the results have been summed to provide energy gain, energy consumption in the case of pump operation, and hot water consumption within one month period.

Solar radiation energy $E_{S O L}$ that is incident on the two sets of solar collector surfaces of the total area $146.29 \mathrm{~m}^{2}$ has been calculated using Eq. (3). The total amount of $E_{S O L}$ from 1 May 2015 to 30 November 2016 was equal 1029.72 GJ.

Next, the amount of useful energy gain coming from both arrays of the solar collectors $E_{S C}$ has been determined based on the data recorded by heat meters placed in the substation on the roof. During the reporting period of 19 months the solar collectors provided 397.98 GJ of thermal energy, of which 204.09 GJ has been produced by flat plate collectors, and 193.89 GJ by evacuated tube collectors. The bar chart in Fig. 3 shows that the ETCs (much more technologically advanced) have been characterized by higher power only in the cold season from October to March. Unfortunately, at this period of the year duration and intensity of solar radiation is very low. Heat losses to the environment from flat plate collectors are usually higher compared to from evacuated tubes. However, the overall balance has shown that FPCs produced 5\% more energy during the whole analysed time period. Part of this energy has been lost. The vast majority of sources of energy loss are outlined on the block diagram shown in Fig. 4. 


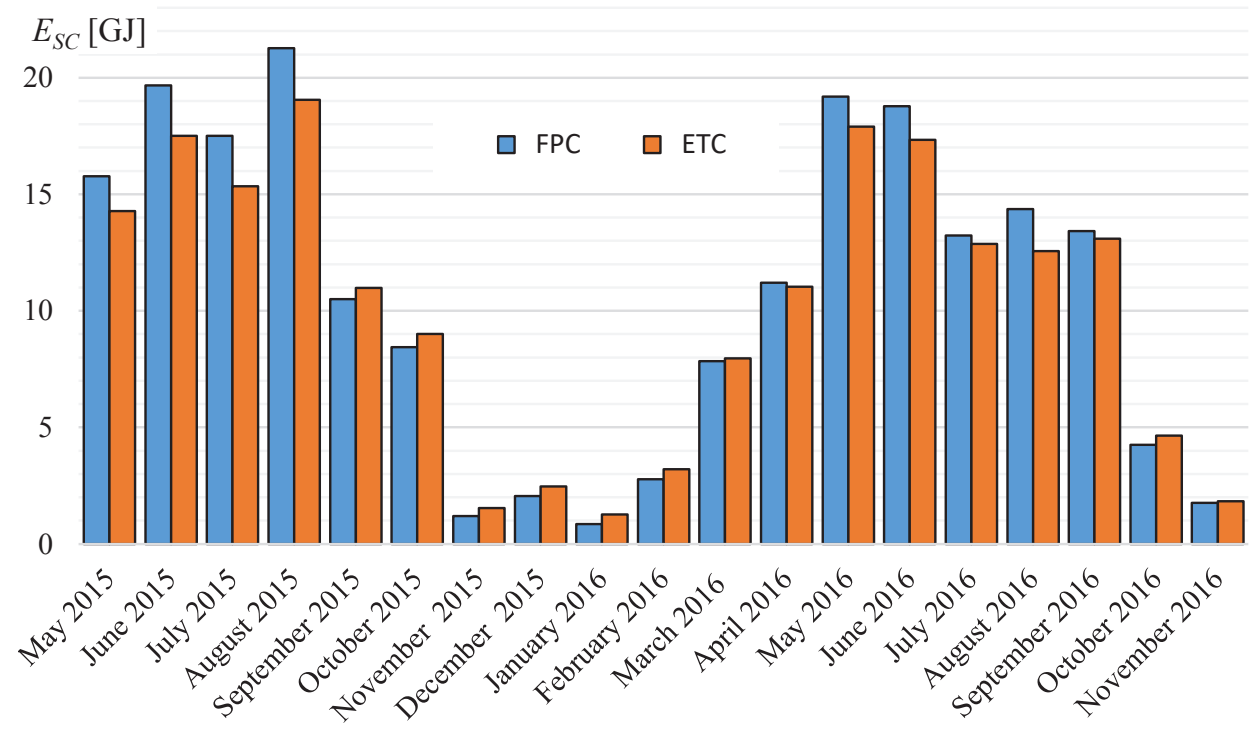

Fig. 3. Solar energy converted into thermal energy by solar panels.

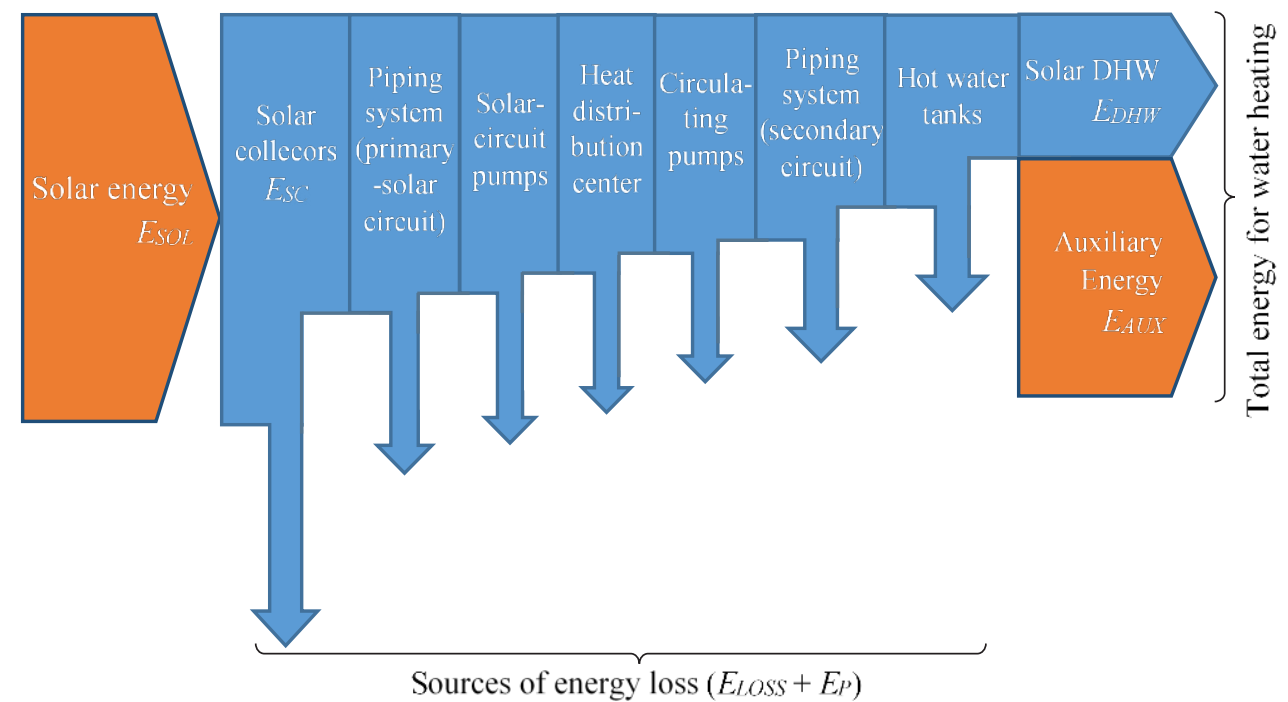

Fig. 4. Energy balance of the SDHW system.

The closed loop solar system under the analysis is divided into primary and secondary circuits, that are separated by heat exchangers. Hence, such a system must be fitted with four pumps, two for each of the collector arrays. All pumps consumed 8.69 GJ of electricity $E_{P}$ in the period under consideration. This value has reduced energy gain from the solar system. The pumps located in the ETCs closed loop system consumed about 5.5\% more of electric energy compared to FPCs system. As can be observed in Fig. 5, this trend intensified during the winter. 


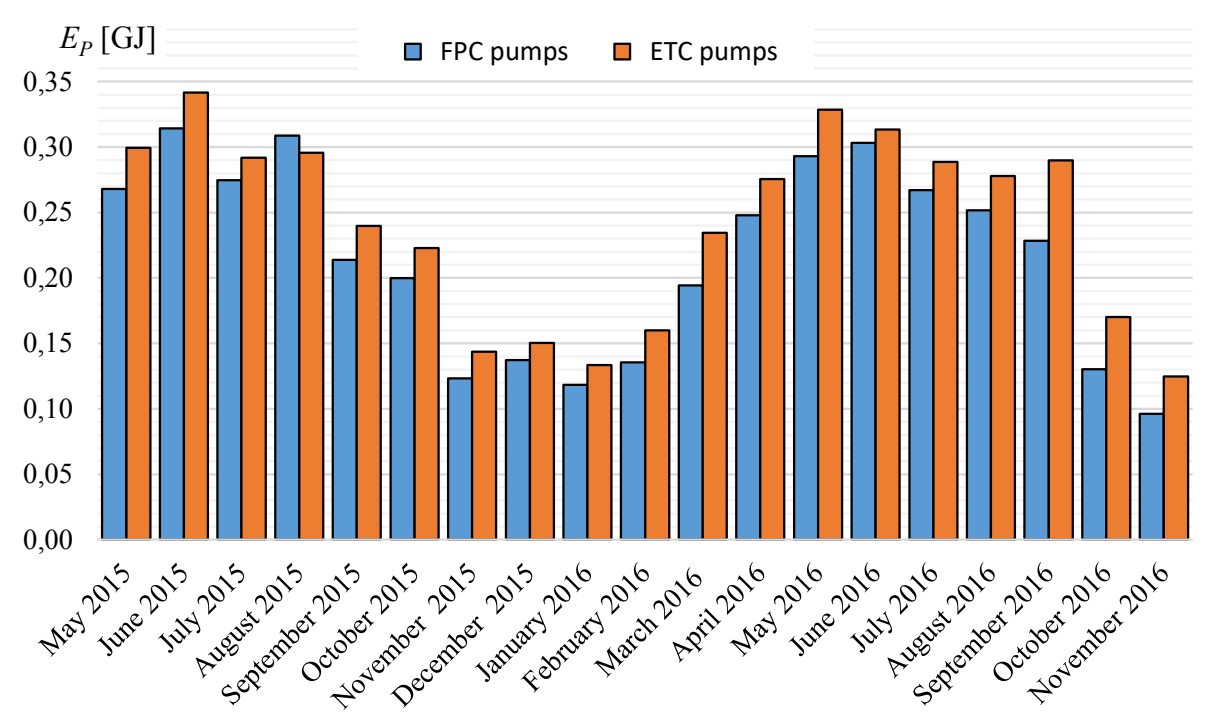

Fig. 5. Consumption of electric energy by the pumps by months.

The useful heat energy supplied to the domestic water system $E_{D H W}$ has been calculated separately for each type of solar panel sub-systems based on Eq. (2) and was equal 344.52 GJ. Then, seasonal energy efficiency of the whole SDHW system has been estimated using the relation given in Eq. (1). Fig. 6 shows the $\eta_{S Y S}$ taken separately for each month during the entire study period. As can be observed, in the warmer months from April to August, the efficiency was the highest and most often exceeded $35 \%$. In the winter $\eta_{S Y S}$ was significantly decreasing and even it dropped up to 10\% in January 2016.

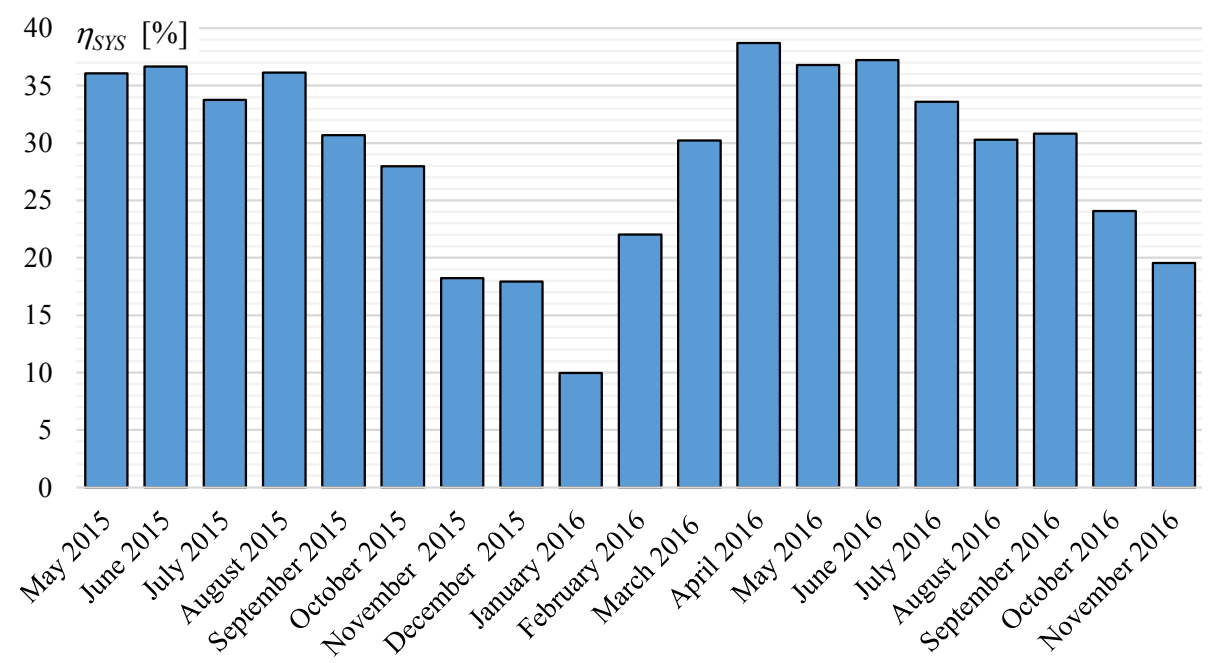

Fig. 6. Energy efficiency of the SDHW system by months.

Eight hot water storage tanks, the hot water pipelines connecting substation on the roof with the second substation in the basement (average length of each of the four DN40 pipes is $86 \mathrm{~m}$ ), flow control fittings and shut-off valves turned out to be the major sources of energy loss $E_{\text {LOSS. }}$. Detailed analysis of the different sources of heat loss was not the main 
subject of this study. They have been taken as a total. The value of $E_{L O S S}$ was equal 53.46 GJ and has been obtained as the difference between $E_{S C}$ and $E_{D H W}$. This was only $13.4 \%$ of energy gain coming from the solar collectors.

The parameter which indicates what part of energy is provided by the solar system and what must be supplied by an additional power source is a solar fraction $S F$. This value is determined by the following formula [8]:

$$
S F=\frac{E_{D H W}}{E_{D H W}+E_{A U X}}
$$

The auxiliary energy demand was calculated according to Eq. (5), assuming the design value for hot water temperature $T_{D H W}$ equal to $60^{\circ} \mathrm{C}$.

$$
E_{A U X}=\sum_{1}^{n m}\left[m_{D H W} \cdot n d \cdot 24 \cdot 3600 \cdot\left(c_{p} \cdot\left(T_{D H W}-T_{F P C}\right)+c_{p} \cdot\left(T_{D H W}-T_{E T C}\right)\right)\right]
$$

As shown in Fig. 7, SDHW system was able to satisfy around 35\% of the hot water demand during the warmer months. However, in winter time, this factor decreased to only about $5 \%$. The monthly average $S F$ in the period under consideration was $23.4 \%$. Usually it is assumed that a well-designed solar system should be able to provide around $40 \%$ of total energy load for water heating [9]. It proves too small area of solar collectors used in this system. Unfortunately, in medium-rise buildings ( 5 to 15 storeys) often there is a lack of space on the roof to allocate additional solar panels.

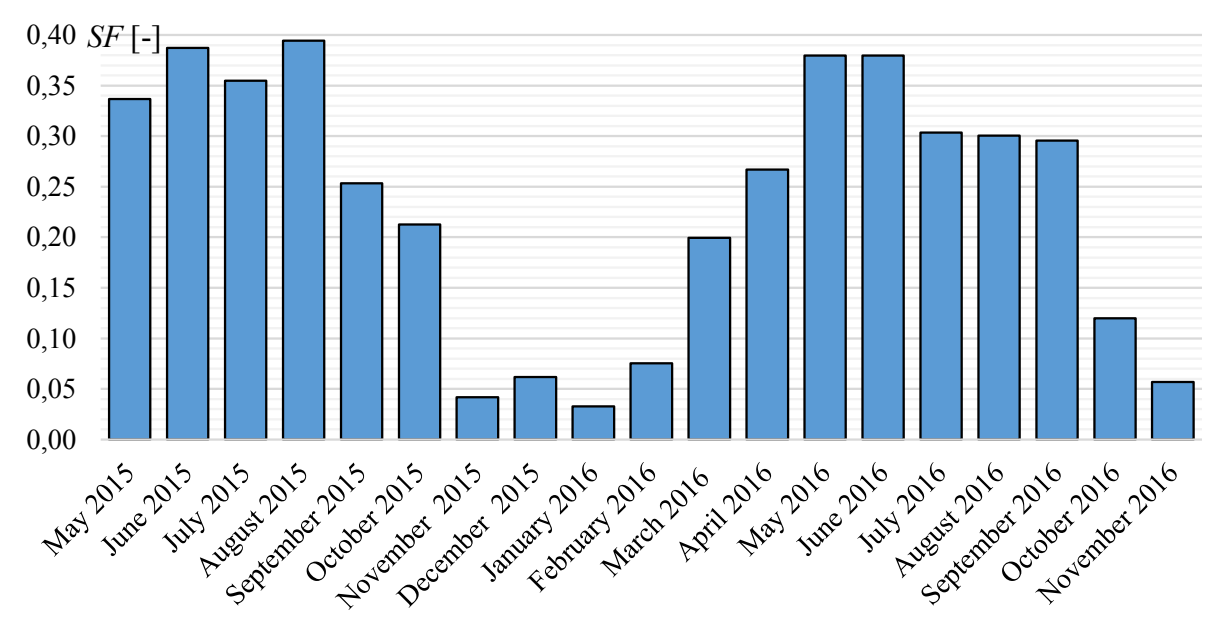

Fig. 7. Changing the monthly average value of the solar fraction.

\section{Conclusions and the future work}

The main goal of the present research has been to determine the seasonal energy efficiency of the solar domestic hot water system located on the campus of Bialystok University of Technology. This study involving analysis of measurement data that have been collected from 1 May 2015 to 30 November 2016.

It has been found that the energy efficiency of SDHW system was strongly related to the intensity of solar radiation. As indicated by the calculation results $\eta_{S Y S}$ was equal $32.6 \%$ 
for the time period of this analysis, with $34.7 \%$ in the warmer months and $24.2 \%$ during the colder months (period from October 2015 to March 2016).

To summarize, it can be concluded that only about $1 / 3$ of the solar energy that falls on the solar panels has been converted into useful heat supplied to the domestic hot water system. So, this level of energy efficiency can be applied to any type of economic evaluations associated with the use of solar collectors for water heating in similar types of buildings in areas with a similar climate to north-eastern Poland.

It is planned that the study on the effectiveness of SDHW system will be continued for the next three years. Besides, the thermal performance of both types of solar collectors according to the standard ISO 9806:2013 will be determined.

This work has been performed within the framework of a Grant of Bialystok University of Technology (Grant No. S/WBIIS/4/2014).

\section{References}

1. C. Yao, B. Hao, S. Liu, X. Chen, Energy Procedia, 70 (2015)

2. J.-H. Yoo, Energy and Buildings, 101 (2015)

3. L. Gill, J.M. Mahon, K. Ryan, Solar Energy, 137 (2016)

4. S. Stevanovic, M. Pucar, Energy and Buildings, 54 (2012)

5. Y. Hang, M. Qu, F. Zhao, Energy and Buildings, 45 (2012)

6. W.M. Lin, K.C. Chang, K.M. Chung, Renewable and Sustainable Energy Reviews, 41 (2015)

7. R. Dziugaite-Tumeniene, V. Motuziene, G. Siupsinskas, K. Ciuprinskas, A. Rogza, Energy and Buildings, 138 (2017)

8. The German Solar Energy Society, Planning and installing solar thermal systems: A guide for installers, architects and engineers, James and James, UK (2007)

9. Y. Evans, T. Dwyer, CIBSE Journal, 02 (2009) 